\title{
A Seamless Approach to Stitching Lunar DOMs with TPS
}

\author{
Hao Sheng ${ }^{1,3}$, Chen Lou ${ }^{2}$, Wujian $\mathrm{Xu}^{2}$, Chao Li ${ }^{1,3 *}$ and Lingli Mou ${ }^{4}$ \\ ${ }^{1}$ School of Computer Science and Engineering, Beihang University, Beijing 100191, China \\ ${ }^{2}$ School of Instrument Science and Opto-electro Engineering, Beihang University, Beijing 100191, China \\ ${ }^{3}$ Shenzhen Key Laboratory of Data Vitalization, Research Institute in Shenzhen, Beihang University, Shenzhen 518057, China \\ ${ }^{4}$ National Astronomical Observatories, Chinese Academy of Sciences, Beijing 100012, China
}

Received: 4 Oct. 2012, Revised: 24 Dec. 2012, Accepted: 27 Dec. 2012

Published online: 1 Jun. 2013

\begin{abstract}
Aiming at the mosaic technology of the orthophoto acquired by Chinese remote sensing satellite CE-1, a thin-plate spline (TPS) function based method of image mosaic is proposed while the traditional mosaic method seems impossible. With the advantages of TPS (Thin-plate Spline) function in image transformer, the image fusion technology is introduced to get the seamless image mosaic result. Considering the mosaic result of lunar DOMs (Digital Orthophoto Map) by CE-1, a sharp gray jump usually exists beside the stitching line. To solve the uneven gray distribution caused by light changing or position difference during photographing, a method is described which is based on dynamic rules to stretch image according to the gray histogram. Using CE-1's $2 \mathrm{C}$ data, the experimental result states that our approach can guarantee the accuracy of the geographical precision and can greatly meet the needs of seamless mosaic with good visual effect.
\end{abstract}

Keywords: seamless mosaic, thin-plate spline, best-stitching curve, edge smoothing

\section{Introduction}

As the $2 \mathrm{C}$ data of CE-1 are stitched by separated images, there are two problems: firstly, obvious gray jump appears near the stitching curve which has negative effects on the continuity of image; secondly, the contrast level may be different from each sections caused by sensor's own factors, such as imaging angle, light condition, imaging location. The interpretation of the images and the subsequent processing are affected. Aiming at the accuracy of the geographical precision and the visual effect, geometric correction and radiometric correction should be done to the images.

For geometric correction, a method including optimal seam searching algorithm and TPS-based transformation is introduced to guarantee the geographical precision of each image. Srinivasa.RB [1] proposed a FFT-based image registration method that can handle pan, zoom and scale changes, but can only be used when images strictly meet the definition of the change relationship. Kim DH et al. [2] achieved image registration by reducing the differences between images' pixel to get the perspective transformation matrix, but the global energy is hard to be solved. Feature-based method using rigid model like ZD space projection model developed by R.szeliski and J.coughlan [3] can fit the images taking in different geometric conditions, but cannot meet the requirement of edge fixed. Non-rigid model such as Polynomial model using a LSM strategy to calculate the transformation function may result in the inaccuracy of coordinates. Thin-plate spline function includes both rigid and non-rigid features, can reach the goal of high accuracy on control points and fitting the overlapping region well.

For radiometric correction, a method based on sliding window and separated weighted fusion was proposed. The common ways used to eliminate image mosaic lines include: 1.wavelet transformation based method [4,5], however it is relatively difficult to realize because of the complicated calculation; 2. overlapping images based method [6], when dealing with mosaic images of poor precision, the effect is not satisfying; 3.gradient theory based method [7], some fuzzy problems would be caused around joining area, and the ability of processing structure dislocation is weak.

In order to overcome the limitations of the traditional feature-based mosaic methods, this paper describes a method to create seamless mosaics based on the modeling of the geometric and radiometric changes. The algorithm

\footnotetext{
*Corresponding author e-mail: licc@ buaa.edu.cn
} 
of best-stitching curve searching and TPS-based transformation solves the problems caused by geometric changes, while a method based on sliding window and separated weighted fusion is introduced to make brightness adjustment and get better visual effect.

\section{Framework}

In this section, the framework of the TPS-based lunar DOMs seamless mosaic method is provided. As shown in Fig.1, the architecture of the method is described as follows:

Firstly, the CE-1 images are obtained and processed by the preprocessing module. In order to enhance the mosaic accuracy, this part contains image grayscale adjustment and resampling.

Secondly, the preprocessed images are transferred to the image mosaic module to get the seamless mosaic result. During this procedure, best-stitching curves searching and TPS-based transformation are applied.

Finally, the method based on sliding window and separated weighted fusion is proposed to eliminate the obvious stitching curve and uneven brightness in the elementary mosaic image.
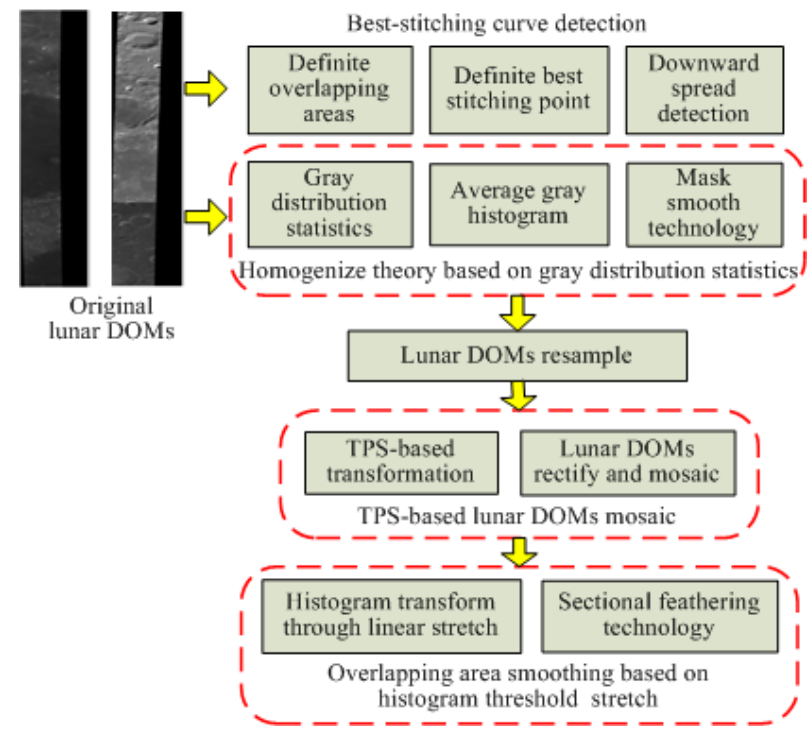

Fig. 1 The overall processing of TPS-based lunar DOMs mosaic

The remainder of this paper is organized as follows. The preprocessing before stitching is described in Section 3 . The TPS-based mosaic module for lunar DOMs is built in Section 4. The edge smoothing method based on histogram threshold stretching and section gray estimate is proposed in Section 5. The experimental results are given in Section 6, and finally, the conclusion is summarized in Section 7.

\section{DOM preprocessing}

DOM preprocessing is the first essential step of building DOMs' mosaics which mainly consists two parts: 1) Homogenization method based on gray statistics. 2) Lunar DOMs resampling.

\subsection{Homogenization method based on gray statistics}

To improve the uneven gray distribution in lunar orthophotos, a homogenization method based on statistics of gray distribution is proposed. Firstly, the average gray distribution is calculated. Secondly, the trend of gray is found by smoothing the statistic results. Finally, homogenize the image gray level according to the trend of gray. Fig. 2 shows the average gray distribution of 200 pixels width image under different masks:

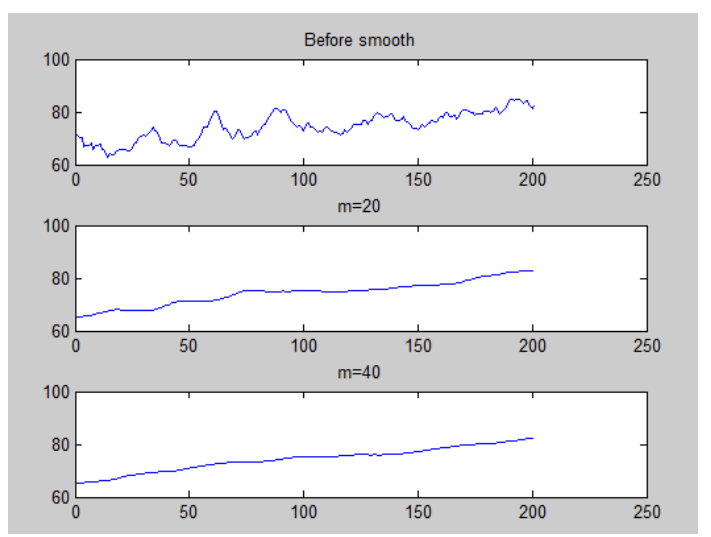

Fig. 2 The statistic results after processing of different mask size

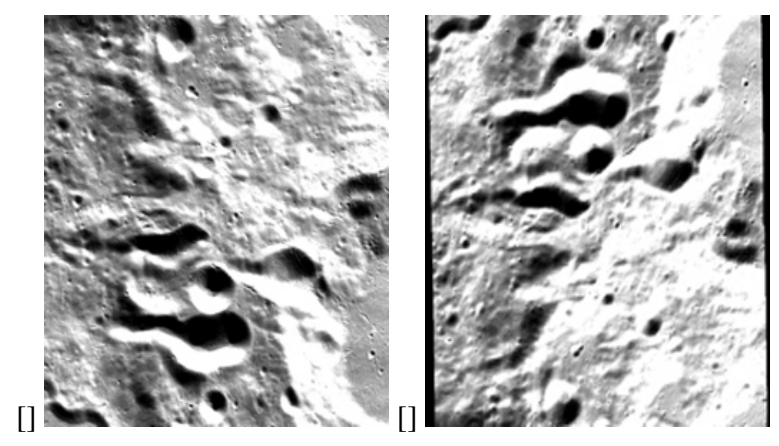

Fig. 3 Original image and UTM projected image 


\subsection{Lunar DOMs resampling}

In order to reduce the geometric differences between DOMs, the projection and resolution of CE-1's DOMs should be unified. By reprojecting the coordinates to the lunar surface projection system and resampling the DOMs to geographic grid, the DOMs with same projection method and resolution are gained. As shown in Fig.3, the projected experimental image is reversed vertically and tiled.

\section{Geometric Correction}

Aiming at the geometric distortion of the adjacent images? overlapping area, the lunar DOMs mosaic method based on best-stitching curve searching algorithm and TPS-based transformation is proposed.

\subsection{TPS-based Image Transformation}

Thin-plate spline is a kind of RBF designed which can be constrained by the control points to perform a physical deformation with minimal bending energy [8]. Therefore, the TPS-based transformation model can create a flexible transformation between two domains smoothly.
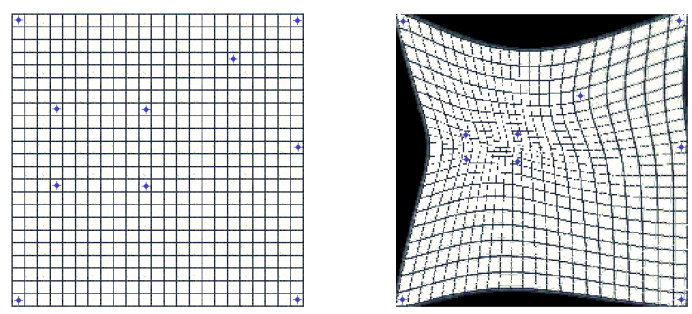

Fig. 4 TPS-based image transformation

As shown in Fig.4, suppose $x_{1}$ and $x_{2}$ to be the corresponding points' coordinates in transformed image and reference image, the dimension is $n \times 2$ :

$$
I=\left[\begin{array}{cc}
K & Q \\
Q^{T} & 0
\end{array}\right]
$$

In the formula, $I$ is the coefficient matrix of the linear equation to solve the transformation matrix $W$, where $K$ is a $n \times n$ matrix. $K_{i j}=U\left(\left|x_{1}^{i}-x_{2}^{j}\right|\right), U(r)=r^{2} \log r$ is the TPS basis function, $Q$ is a $n \times 3$ matrix $Q=\left[1, x_{1}\right]$.

Given $X^{\prime}=\left[x_{2}, 0,0,0\right]^{T}$, the linear equation can be formed as below, where $W$ is the transformation matrix:

$$
I^{T} W^{T}=X^{\prime} .
$$

By using LU decomposition method to solve the linear equations, the transformation matrix $W$ is obtained. For any point $x$ in the transformed image, the pixel coordinates after TPS transformation is:

$$
\begin{gathered}
x^{\prime}=W U_{d}(x) . \\
U_{d}(x)=\left(U\left(x-x_{1}\right), U\left(x-x_{2}\right), \ldots U\left(x-x_{n}\right), 1, x^{T}\right) .
\end{gathered}
$$

Based on the TPS function, the transformation between two images is established by the constraints of control points. In order to obtain a complete image transformation without gaps, a reversed mapping method is used to create the transformation result that reduces the computational cost.

\subsection{Best-stitching curve searching algorithm}

To guarantee the coordinates precision, the basic rule of stitching curve searching algorithm is to make changes to the original image as few as possible. By considering the relative relationship of two adjacent images, the initial stitching curve can be created. Finally, a blocked SURF feature matching algorithm is applied to find the final stitching curve. The best-stitching curve searching algorithm proposed above includes three steps:

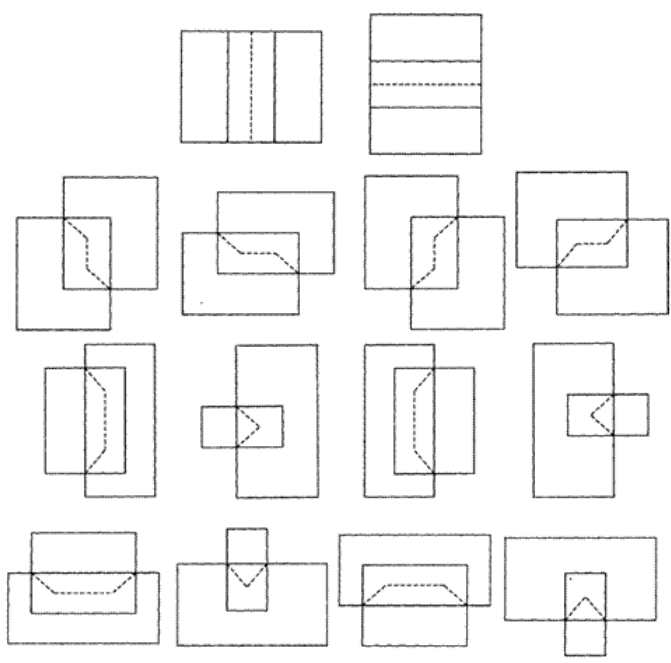

Fig. 5 Types of initial stitching curve

1. Create the initial stitching curve. Every stitching curve begins and ends with an angle bisector of the overlapping area. There are 14 kinds of relative positions shown in Fig. 5 taken into consideration.

2. Find corresponding points through a blocked SURF feature matching method. In order to ensure the uniform distribution of the matching points in overlapping area, a sliding window moving along the initial stitching curve is 
used to find the corresponding points. The numbers of matching points need to be limited by the distance threshold between the initial stitching curve and the points. RANSAC filter is used to detect the error matching points.

3. Generate the final stitching curve. After the error matching points are eliminated by RANSAC filter [9], the midpoints of every two corresponding points are set as control points, which form the final stitching curve sorted in horizon or vertical direction.

\subsection{TPS-based DOMs mosaic method}

As stated above, the results of TPS transformation are constrained by control points. The points on the edge of overlapping regions are seen as fixed points, and the corresponding points are taken as the original points. Both of them are used to ensure the consistency of geometry together.

The details of the proposed method are given as follows: Firstly, the fixed points are selected by sampling along the overlapping areas' edges with a certain step length, while the control points are defined as the midpoints of two corresponding points. Secondly, the overlapping area of two DOMs is transformed with the TPS-based mosaic model determined by the fixed points, corresponding points and original points. Finally, the overlapping area is divided into two parts by the stitching curve and then filled with the pixel value from two DOMs separately.

\section{Radiometric Correction}

During the radiometric correction, an edge smoothing method based on histogram threshold stretch will be adopted. On the basis of fulfilling the overlapping area in geometric correction, the image gray histogram of both sides of the stitching curve can be generated. In order to make the two parts of the image unified to the same gray and contrast level, linear stretching will be applied. Firstly, according to different weighted gray level in the histogram, choose proper threshold value. Set the gray value which reaches $\lambda_{\min }$ and $\lambda_{\max }$ percentage of the total pixel number as the stretch threshold. Suppose the chosen threshold values are $\min$ and $\max$, the formula of stretching is as follows:

$$
p_{x y}^{\prime}=\frac{p_{x y}-\min }{\max -\min } \times 255 .
$$

According to the statistical histogram information, stretch the gray image is stretched using proper threshold value $\lambda_{\min }$ and $\lambda_{\max }$, so that the gray level and contrast standard of adjacent images can be unified.

After finishing the processes above, the gray level and contrast standard of images in target area may be nearly the same. To achieve better effects, the feathering method is applied. According to the differences between pixels on the two sides of the stitching curve, variable ranges will be chosen to adjust the gray value so that the image is visually smooth.

\section{Experiments and Results}
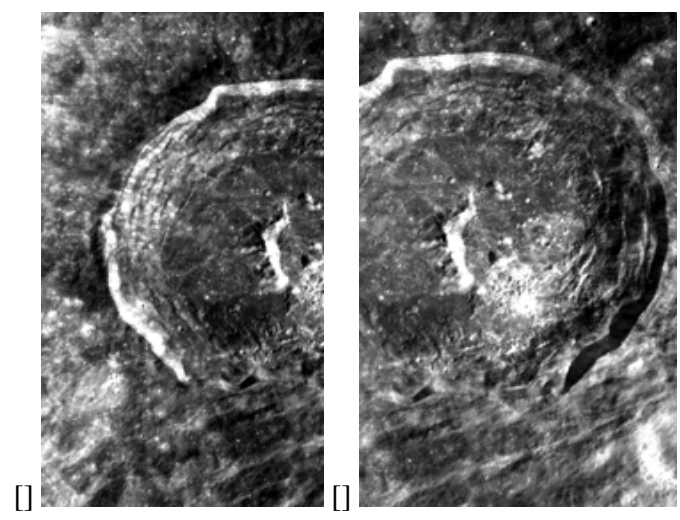

Fig. 6 Lunar 2C DOMs data of CE-1

In this section, the proposed TPS-based method is used to stitch lunar DOMs. Taking the $2 \mathrm{C}$ data of CE-1 as the primitive experimental data, the interested areas covered lunar latitude range between $66.68138098^{\circ} \mathrm{N}$ and $75.13940992^{\circ} \mathrm{S}$, and longitude range between 54.11036640 $\mathrm{E}$ and $62.45546389^{\circ} \mathrm{E}$. As shown in Fig.6, the DOMs are the part of the interested areas.
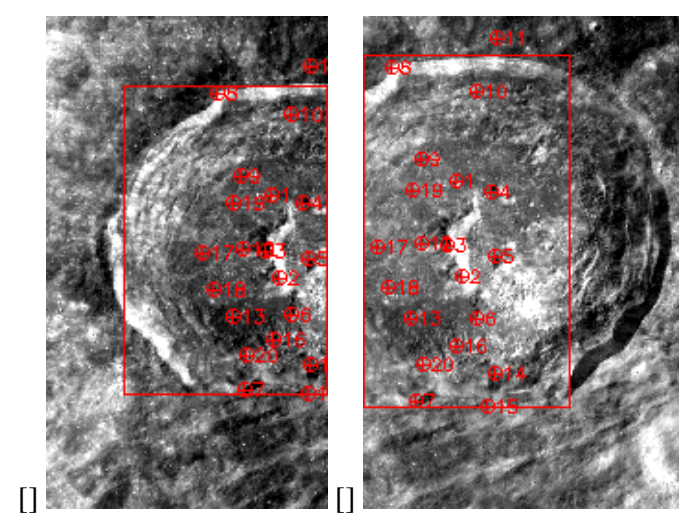

Fig. 7 The results of feature matching 


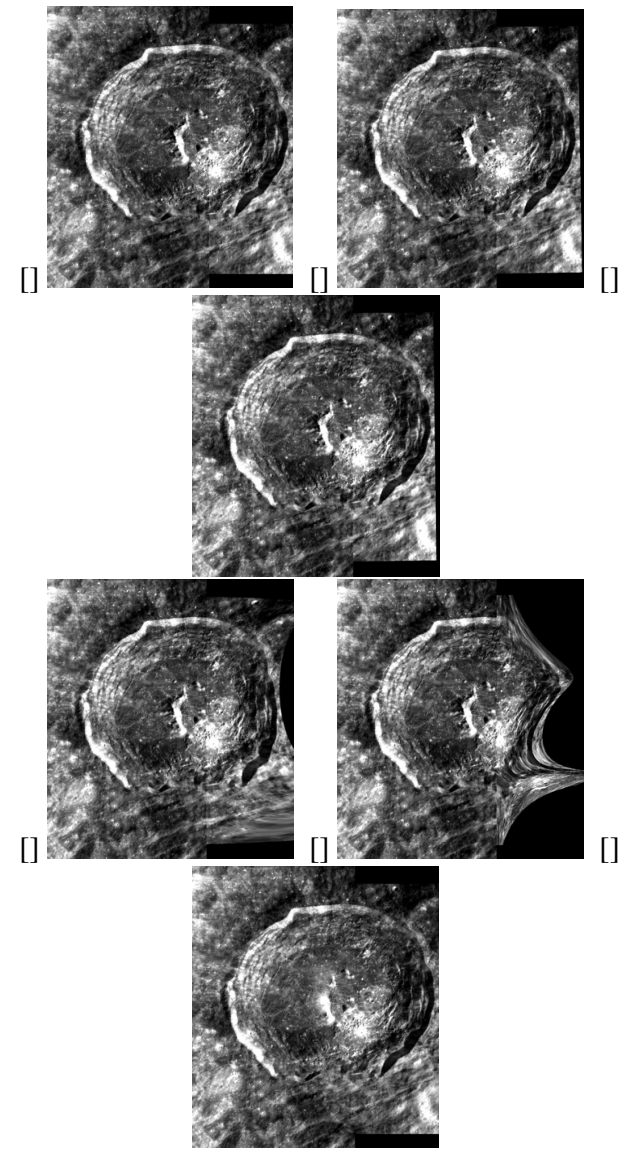

Fig. 8 Detail of the mosaic results. (a) Reference image; (b) Affine; (c) Perspective; (d) 2 order polynomial; (e) 3 order polynomial; (f) TPS-based model

\subsection{Mosaic Results and Discussion}

In Fig.7, the matching points in the overlapping regions are extracted by Surf feature. Based on the results of Surf feature matching, different mosaic methods are applied such as affine transformation, perspective transformation, polynomial transformation, and TPS-based method. The mosaic results of different mosaic methods are shown in Fig.8.

As Fig. 8 demonstrate the polynomial transformation leads to large geometric distortion with same amounts of control points. The affine transformation, perspective transformation and TPS-based method restore the relative position of two DOMs nearly the same with the reference DOM.

From Fig.9, the results of affine transformation and 2 order polynomial transformation show obvious malposition along the stitching curve. In order to quantifying the evaluation of stitching effect, 60 points along the perpendicular direction of the stitching curve

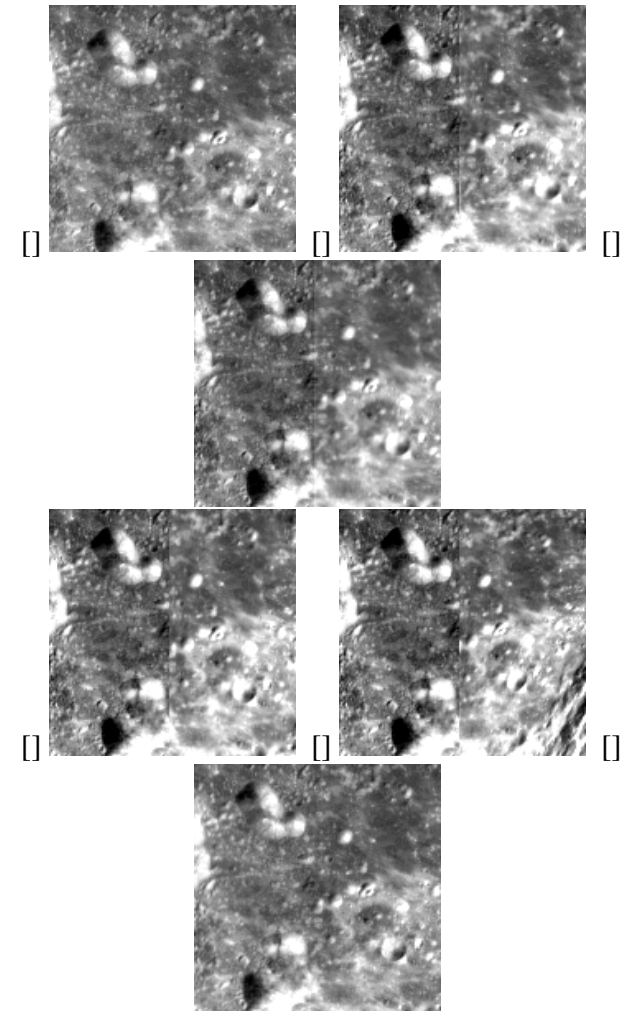

Fig. 9 Detail of the mosaic results. (a) Reference image; (b) Affine; (c) Perspective; (d) 2 order polynomial; (e) 3 order polynomial; (f) TPS-based model

(on both sides, each 30 pixels) are selected to make the gray histograms shown in Fig. 10.

\begin{tabular}{|c|c|}
\hline Mosaic methods & Similariy \\
\hline affine transform & 0.3304 \\
\hline perspective transform & 0.4459 \\
\hline 2 order polynomial & 0.4709 \\
\hline 3 order polynomial & 0.4821 \\
\hline TPS-based model & 0.9989 \\
\hline
\end{tabular}

Table 1 Similarity of curves with different mosaic methods

To estimate the similarity between the transformed DOM and the reference DOM, the curve similarity is measured by Clark's spectral curve fitting theory [10]. The degree of similarity $F$ is presented as below:

$$
F=\frac{n \sum O_{c} L_{c}-\sum O_{c} \sum L_{c}}{\sqrt{\left[n \sum O_{c}^{2}-\left(\sum O_{c}\right)^{2}\right]\left[n \sum L_{c}^{2}-\left(\sum L_{c}\right)^{2}\right]}} .
$$

where $O_{c}$ is the $n$ pixel gray value of the reference DOM and $L_{c}$ is the $n$ pixel gray value of the transformed DOM. 


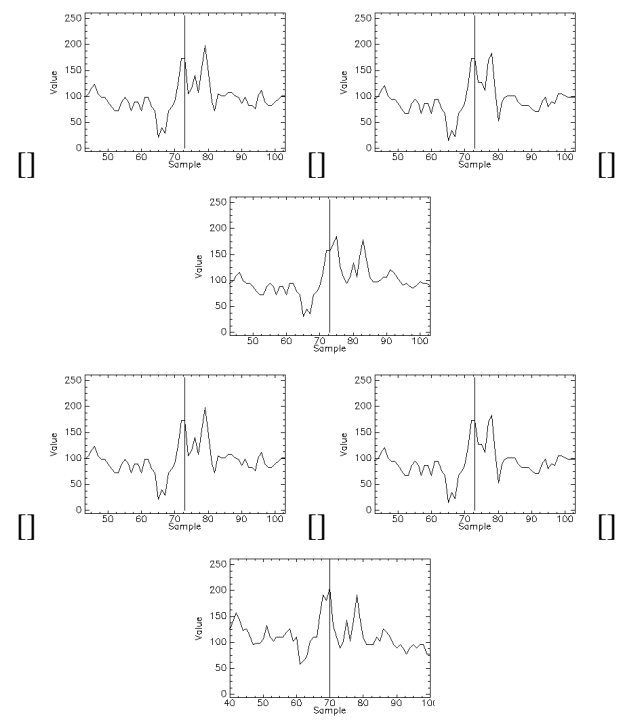

Fig. 10 Total mosaic results. (a) Reference image; (b) Affine; (c) Perspective; (d) 2 order polynomial; (e) 3 order polynomial; (f) TPS-based model

Table. 1 summarizes the similarity of curves with different mosaic methods.

Compared with the similarity of curve, the result carried out by TPS-based mosaic method maintains more similarity with the reference image. By TPS-based method, the high coordinates? accuracy of mosaic can be achieved.

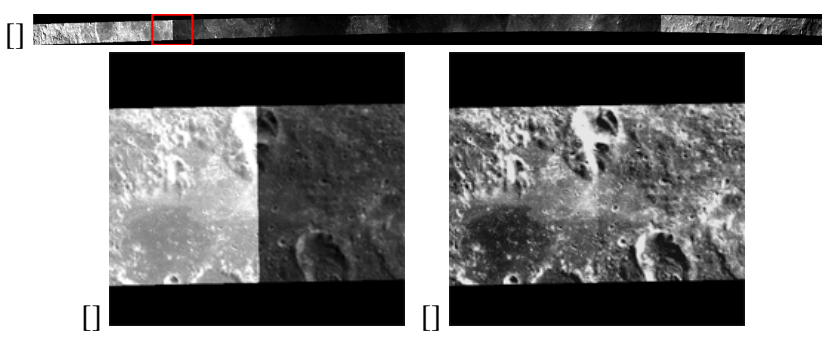

Fig. 11 The homogenized result of the stitching curve. (a)Original DOM; (b)The stitching curve; (c)The homogenized result

\subsection{Fusion Results and Discussion}

From Fig.11(a), the obvious gray jump can be found near the stitching curve. Fig.11(b) shows the area with obvious gray jump near the stitching curve. By using the histogram threshold gray stretch, the brightness and contrast of DOMs on both sides of the stitching curve will be homogenized to similar standard, as is shown in Fig.11(c).

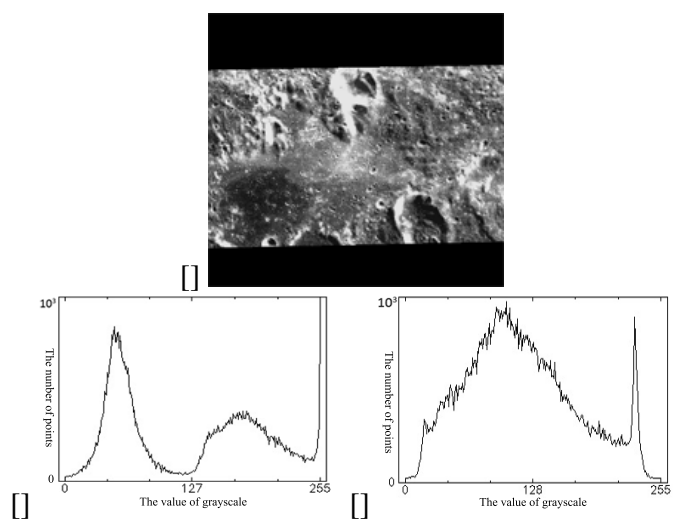

Fig. 12 The result of the stitching curve elimination

The final result of the stitching curve elimination using the feathering method is shown in Fig.12(a). Fig.12(b) include two obvious wave crests, which reflected the brightness difference in Fig.12(b). After fusion processing, the histogram was normalized.

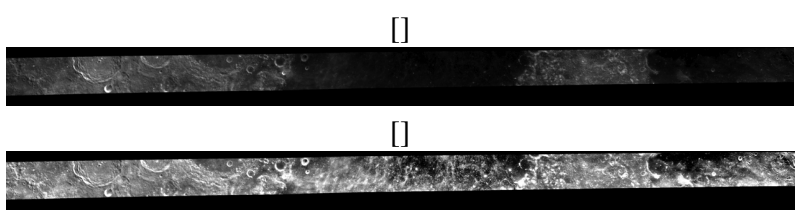

Fig. 13 The final result of fusion

In the experiment, the reference value $C$ was set to 155 . As shown in Fig.13(b), the even gray distribution is gained after homogenization.

However, the change of contrast was uncertain in the process of homogenization. Unifying the contrast and gray level to a same reference value $C$, the stitching curve and uneven distribution of brightness and contrast could transit smoothly. At the same time, the details could be enhanced.

\section{Conclusion}

This paper presents a seamless mosaic approach for lunar DOM based on TPS. Surf features are used to eliminate the suitable stitching curve for the transformation model. After the TPS function is embedded in the mosaic method, TPS-based transformation on the overlapping regions maintains high geographical precision of mosaic. Further, in order to reduce the uneven distribution of 
brightness and contrast caused by mosaic and photographing, the method of stitching curve elimination and edge smoothing based on histogram threshold stretch is presented. The fusion technology homogenizes the brightness and contrast level based on statistics of partial gray distribution. Experimental results in real lunar DOMs by CE-1 show that our approach outperforms others in seamless mosaic of DOMs. The results of mosaic prove that our approach can guarantee the accuracy of the geographical precision and can greatly meet the needs of seamless mosaic with a good visual effect.

\section{Acknowledgement}

This work was partially supported by the Natural Science Foundation of China (NSFC) under grant \#61103094, the National High Technology Research and Development Program of China (No.2011AA010502), the National Science \& Technology Pillar Program (2012BAH07B01), and the Open Fund of the State Key Laboratory of Software Development Environment under grant \#SKLSDE-2012ZX-04.

\section{References}

[1] R. Szeliski and J. Coughlan, Hierarchical spline-based image registration. IEEE Computer Society Conference on Computer Vision and Pattern Recognition, 194-201 (1994).

[2] D.H Kim, Y.I Yoon, and J. S Choi, An effcient method to build panoramic image mosaics. Pattern Recognition Letters 24(14), 2421-2429 (2003).

[3] R.B Srinivasa, and B.N Chatterji, An FFT-based technique for translation,rotation, and scale-invariant image registration. IEEE Transactions on Image Processing 5(8), 1266-1271 (1996).

[4] L.J Chipman, T.M Orr, and L.N Graham, Wavelets and image fushion. International Conference on Image Processing 3, 248-251 (1995).

[5] J. Nunez, X. Otazu, O. Fors, A. Prades, V. Pala, R. Arbiol, Multiresolution-based image fusion with additive wavelet decomposition. IEEE Transactions on Geoscience and Remote Sensing 37(3), 1204-1211 (1999).

[6] S. Peleg, Elimination of seams from photomosaics. Computer Graphics and Image Processing 16(1), 90-94 (1981).

[7] G. Shiming, C. Yimin, L. Jie, and Z. Ling, A gradient field based seam elimination method. Journal of Computer-Aided Design \& Computer Graphics 19(2), 227-232 (2007).

[8] G. Wu, Q. Wang, H. Jia, D. Shen, Feature-based groupwise registration by hierarchical anatomical correspondence detection. Human Brain Mapping 33(2), 253-271 (2012).

[9] C. Ondrej and M. Jiri, Optimal randomized ransac. IEEE Transactions on Pattern Analysis and Machine Intelligence 30(8), 1472-1482 (2008).

[10] C. Clark, and A. F. Clark, Spectral identification by singular value decomposition. International Journal of Remote Sensing 19(12), 2317-2329 (1998).
Hao Sheng received his $\mathrm{BSc}$ and $\mathrm{PhD}$ from the School of Computer Science and Engineering of Beihang University, Beijing, China in 2003 and 2009, respectively. Now he is assistant professor at Beihang University. He is working on computer vision, intelligent transportation systems, and hyperspectral remote sensing.

\section{Chen}

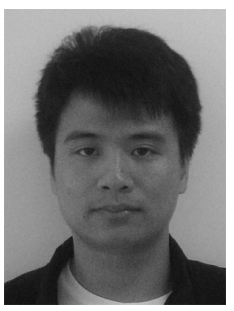
Lou, born in 1987, $\mathrm{PhD}$ candidate of Beihang University. His research interests include image processing and remote sensing imaging simulation.

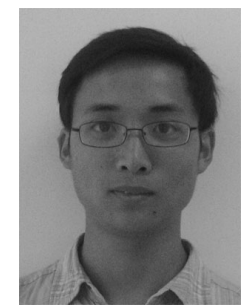

Wujian Xu, born in 1987, PhD candidate of Beihang University. His research interests covered polarization navigation system and image processing. 
Chao Li received his $\mathrm{BSc}$ and

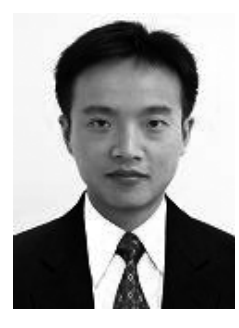
$\mathrm{PhD}$ degrees in computer science and technology from Beihang University, Beijing, China in 1996 and 2005, respectively. Now he is an associate professor in the School of Computer Science and Engineering, Beihang University. Currently, he is working on data vitalization and computer vision. Corresponding author of this paper.

Lingli Mou, born in 1977, Associate Researcher of National Astronomical Observatories, Chinese Academy of Sciences. His research interests covered lunar cartography and spatial information processing. 